University of Wollongong

Research Online

Australian Institute for Innovative Materials -

Papers

Australian Institute for Innovative Materials

2008

Dielectric, magnetic, and magnetotransport properties in Sr doped twodimensional RE2CoO4 (RE=Pr,Eu) compounds

Qiwen Yao

University of Wollongong, qy75@uow.edu.au

Xiaolin Wang

University of Wollongong, xiaolin@uow.edu.au

SX. Dou

University of Wollongong, shi@uow.edu.au

Follow this and additional works at: https://ro.uow.edu.au/aiimpapers

Part of the Engineering Commons, and the Physical Sciences and Mathematics Commons

Research Online is the open access institutional repository for the University of Wollongong. For further information contact the UOW Library: research-pubs@uow.edu.au 


\title{
Dielectric, magnetic, and magnetotransport properties in Sr doped two- dimensional RE2CoO4 (RE=Pr,Eu) compounds
}

\author{
Abstract \\ In this work, we report on the studies of dielectric, magnetic, and magnetotransport properties of $\mathrm{Sr}$ \\ doped $\mathrm{RE}_{2-\mathrm{x}} \mathrm{Sr}_{\mathrm{x}} \mathrm{CoO}_{4}(\mathrm{RE}=\mathrm{Pr}$ or $\mathrm{Eu}, \mathrm{x}=0.25-1.5)$. These compounds were systematically studied in terms of \\ structure, magnetic, magnetotransport, and dielectric constant measurements. Rietveld refinement \\ indicated that these compounds crystallized in $\mathrm{K}_{2} \mathrm{NiF}_{4}$-type structure with space group $14 / \mathrm{mmm}$. Lattice \\ parameters increase with $\mathrm{Sr}$ doping level. The system changes from paramagnetic to ferromagnetic with \\ increasing Sr doping level and finally becomes ferromagnetic with $\mathrm{T}_{\mathrm{C}}$ of $230 \mathrm{~K}$ for $\mathrm{Pr}_{0.75} \mathrm{Sr}_{1.25} \mathrm{CoO}_{4}$ and \\ EuSrCoO 4 , respectively. The temperature dependence of resistivity indicates that both systems change \\ from semiconductive to metallic with Sr doping. The magnetoresistance (MR) value of $10 \%$ at $5 \mathrm{~K}$ and $8 \mathrm{~T}$ \\ is found for the $\mathrm{EuSrCoO}_{4}$ compound. Large dielectric constants with values of above 2000 were \\ observed in low frequencies for samples with $\mathrm{x}$ around 1 for Pr based compounds. \\ Keywords \\ Dielectric, magnetic, magnetotransport, properties, doped, two, dimensional, RE2CoO4, compounds \\ Disciplines \\ Engineering | Physical Sciences and Mathematics

\section{Publication Details} \\ Yao, Q, Wang, X \& Dou, SX (2008), Dielectric, magnetic, and magnetotransport properties in Sr doped two- \\ dimensional RE2CoO4 (RE=Pr,Eu) compounds, In 52nd Annual Conference on Magnetism and Magnetic \\ Materials, NOV 05-09, 2007, Tampa, Florida, USA, Journal of Applied Physics, 103(7), pp. 1-7.
}

This journal article is available at Research Online: https://ro.uow.edu.au/aiimpapers/258 


\title{
Dielectric, magnetic, and magnetotransport properties in $\mathrm{Sr}$ doped two-dimensional $\mathrm{RE}_{2} \mathrm{CoO}_{4}(\mathrm{RE}=\mathrm{Pr}, \mathrm{Eu})$ compounds
}

\author{
Q. W. Yao, X. L. Wang, ${ }^{\text {a) }}$ and S. X. Dou \\ Institute for Superconducting and Electronic Materials, University of Wollongong, Northfields Ave., \\ New South Wales 2522, Australia
}

(Presented on 7 November 2007; received 11 September 2007; accepted 13 October 2007; published online 28 January 2008)

\begin{abstract}
In this work, we report on the studies of dielectric, magnetic, and magnetotransport properties of $\mathrm{Sr}$ doped $\mathrm{RE}_{2-x} \mathrm{Sr}_{x} \mathrm{CoO}_{4}(\mathrm{RE}=\mathrm{Pr}$ or $\mathrm{Eu}, x=0.25-1.5)$. These compounds were systematically studied in terms of structure, magnetic, magnetotransport, and dielectric constant measurements. Rietveld refinement indicated that these compounds crystallized in $\mathrm{K}_{2} \mathrm{NiF}_{4}$-type structure with space group $I 4 / \mathrm{mmm}$. Lattice parameters increase with $\mathrm{Sr}$ doping level. The system changes from paramagnetic to ferromagnetic with increasing $\mathrm{Sr}$ doping level and finally becomes ferromagnetic with $T_{C}$ of $230 \mathrm{~K}$ for $\mathrm{Pr}_{0.75} \mathrm{Sr}_{1.25} \mathrm{CoO}_{4}$ and $\mathrm{EuSrCoO}_{4}$, respectively. The temperature dependence of resistivity indicates that both systems change from semiconductive to metallic with $\mathrm{Sr}$ doping. The magnetoresistance (MR) value of $10 \%$ at $5 \mathrm{~K}$ and $8 \mathrm{~T}$ is found for the $\mathrm{EuSrCoO}_{4}$ compound. Large dielectric constants with values of above 2000 were observed in low frequencies for samples with $x$ around 1 for Pr based compounds. (C) 2008 American Institute of Physics.
\end{abstract}

[DOI: $10.1063 / 1.2830797]$

\section{INTRODUCTION}

Compounds with the $\mathrm{K}_{2} \mathrm{NiF}_{4}$-type structure are well known to exhibit various intriguing physical phenomena, such as high-temperature superconductivity in cuprates, spintriplet superconductivity in ruthenates, and spin/charge stripes in nickelates and manganites. The discovery of superconductivity and metallic ferromagnetism in $\mathrm{Na}_{x} \mathrm{CoO}_{2}^{*} \mathrm{H}_{2} \mathrm{O}$ (Ref. 1) and $\mathrm{Sr}_{2} \mathrm{CoO}_{4}$ has generated a great interests in the study of the two-dimensional $\mathrm{CoO}_{2}$ layer structured compounds. The $\mathrm{K}_{2} \mathrm{NiF}_{4}$-type $\mathrm{Sr}_{2} \mathrm{CoO}_{4}$ consists of $\mathrm{CoO}_{2}$ planes separated by rocksalt-type $\mathrm{SrO}$ planes. It has been reported that the $\mathrm{Sr}_{2} \mathrm{CoO}_{4}$ single-crystalline thin films and polycrystalline samples are metallic ferromagnets with a fairly high Curie temperature $\left(T_{C}\right)$ of $255 \mathrm{~K}$, appreciable magnetic anisotropy, and quasi-two-dimensional transport properties. ${ }^{2,3}$ It has been proposed that $\mathrm{CoO}_{2}$ layers can act as a stage for both spintronic functionality as well as superconductivity. ${ }^{3}$ The valence of the $\mathrm{Co}$ is $4+\mathrm{Sr}_{2} \mathrm{CoO}_{4}$ and makes the $\mathrm{CoO}_{2}$ layer very conductive in the same way as that in $\mathrm{SrCoO}_{3}$ three dimensional perovskite compounds. When the $\mathrm{Sr}$ is fully replaced by rare earth elements (namely, rare earth based $\mathrm{RE}_{2} \mathrm{CoO}_{4}$ system), the compound still remains as $\mathrm{K}_{2} \mathrm{NiF}_{4}$ with two-dimensional $\mathrm{CoO}_{2}$ layers. In this case, the Co becomes 2+ and makes the system insulative or semiconductive and paramagnetic. This has been reflected in the studies of the $\mathrm{Y}$ doped $\mathrm{Sr}_{2-y} \mathrm{Y}_{y} \mathrm{CoO}_{4}$ synthesized under high pressure. ${ }^{2}$ The $\mathrm{Y}$ doping effect indicated that the $T_{C}$ decreases from $255 \mathrm{~K}$ for $y=0$ to $150 \mathrm{~K}$ for $y=0.5$, and ferromagnetism was not observed for $y \geqslant 0.67 .^{2}$ It would be interesting to see how the physical properties of the RE based $\mathrm{CoO}_{2}$ layer structured $\mathrm{K}_{2} \mathrm{NiF}_{4}$-type compounds can be

\footnotetext{
${ }^{\text {a) }}$ Author to whom the correspondence should be addressed. Electronic mail: xiaolin@uow.edu.au.
}

changed by the Sr doping. In this work, we report on the structures, magnetotransport, and dielectric and magnetic properties in $\mathrm{RE}_{2-x} \mathrm{Sr}_{x} \mathrm{CoO}_{4}(\mathrm{RE}=\mathrm{Pr}$ or $\mathrm{Eu})$ compounds.

\section{EXPERIMENT}

Polycrystalline samples $\mathrm{RE}_{2-x} \mathrm{Sr}_{x} \mathrm{CoO}_{4}(\mathrm{RE}=\mathrm{Pr}$ or $\mathrm{Eu}$, $0.25 \leqslant x \leqslant 1.75$ ) were synthesized by conventional solid-state reaction method. Highly pure powders of $\mathrm{Pr}_{6} \mathrm{O}_{11}, \mathrm{Eu}_{2} \mathrm{O}_{3}$, $\mathrm{SrCO}_{3}$, and $\mathrm{Co}_{3} \mathrm{O}_{4}$ were mixed according to appropriate atomic ratios. Samples were sintered in air at $1000{ }^{\circ} \mathrm{C}$ for $12 \mathrm{~h}$ with several intermediate grindings. The powder $\mathrm{x}$-ray diffraction measurements were carried out on a Phillips PW1730 model diffractometer using $\mathrm{Cu} K \alpha$ radiation, and the Rietveld refinement method was applied using the RIETICA program. ${ }^{4}$ Magnetic and transport properties were measured using a commercial Quantum design magnetic property measurement system (MPMS) and physical property measurement system (PPMS) between 5 and $330 \mathrm{~K}$ in magnetic fields up to $8 \mathrm{~T}$. Dielectric constant and loss were measured using a HP 4194A impendence analyzer in the range from 200 up to $10 \mathrm{MHz}$.

\section{RESULTS AND DISCUSSION}

According to the XRD results, it is found that the $\mathrm{Sr}$ has higher solubility in the $\mathrm{Pr}_{2} \mathrm{CoO}_{4}$ system than in the $\mathrm{Eu}_{2} \mathrm{CoO}_{4}$ system. We have found that single 214 phase can be achieved for $x=0.75-1.5$ in $\operatorname{Pr}_{2-x} \mathrm{Sr}_{x} \mathrm{CoO}_{4}$. However, single phase samples can only be formed for $x=1-1.25$ for $\mathrm{Eu}_{2-x} \mathrm{Sr}_{x} \mathrm{CoO}_{4}$ system. Three typical XRD patterns for Pr and Eu based systems are shown in Fig. 1. It can be seen that all the diffraction peaks in the $\mathrm{Eu}_{0.75} \mathrm{Sr}_{1.25} \mathrm{CoO}_{4}$ and $\mathrm{PrSrCoO}_{4}$ belong 


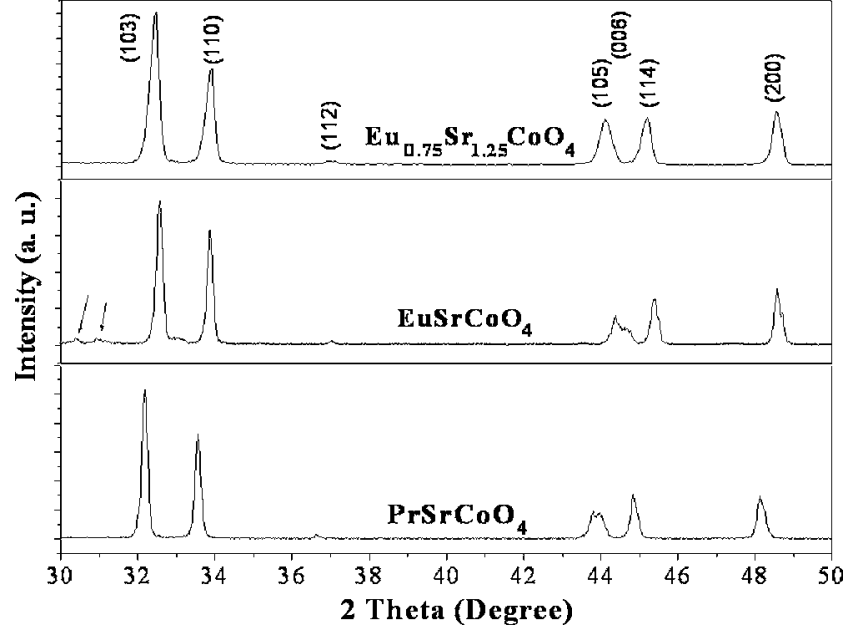

FIG. 1. X-ray diffraction patterns for $\mathrm{RE}_{2-x} \mathrm{Sr}_{x} \mathrm{CoO}_{4}$.

to the 214 phase. Two additional unknown tiny reflections are seen in the $\mathrm{EuSrCoO}_{4}$ compound at around $30.15^{\circ}$ and $30.25^{\circ}$.

The lattice parameters were obtained from the Rietveld refinement using RIETICA program. It was found that the calculated $c$ lattice parameters for both Pr and Eu systems increases with Sr doping level, while the $a$ lattice parameters do not show significant changes. The increasing of the lattice parameters $c$ with Sr doping level is in agreement with the fact that the sizes of $\mathrm{Pr}^{4+}, \mathrm{Pr}^{3+}$, or $\mathrm{Eu}^{3+}$ are smaller than that of $\mathrm{Sr}^{2+}$.

The temperature dependence of the field cooled and zero field cooled dc magnetization for five samples measured at $0.2 \mathrm{~T}$ is shown in Fig. 2. Generally, the $\mathrm{Sr}$ doping changes both systems from paramagnetic to ferromagnetic and enhances the magnetization. Inset shows the inverse susceptibility $\left(\chi^{-1}\right)$ versus temperature for $\operatorname{Pr}_{0.75} \mathrm{Sr}_{1.25} \mathrm{CoO}_{4}$. It reveals a ferromagnetic transition with a $T_{C}$ of about $230 \mathrm{~K}$ for this sample (at the point where the concavity of the graph changes). The $T_{C}$ drops with decreasing Sr content and eventually becomes paramagnetic for $x=0.5$ for the Pr based compounds.

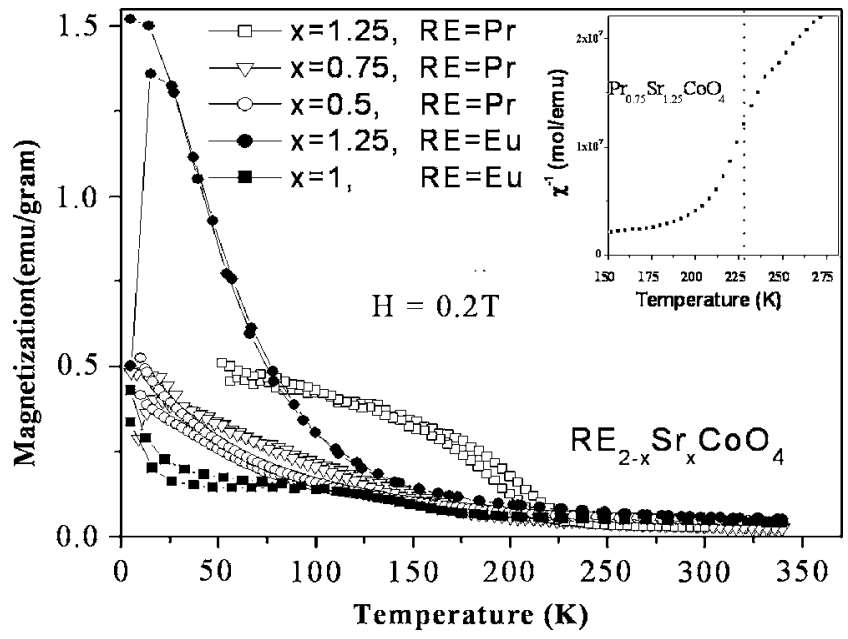

FIG. 2. Temperature dependence of the zero field cooled and field cooled magnetization. Inset shows the inverse susceptibility $\left(\chi^{-1}\right)$ vs temperature for $\mathrm{Pr}_{0.75} \mathrm{Sr}_{1.25} \mathrm{CoO}_{4}$, it reveals a $T_{C}$ of about $230 \mathrm{~K}$ for this sample.

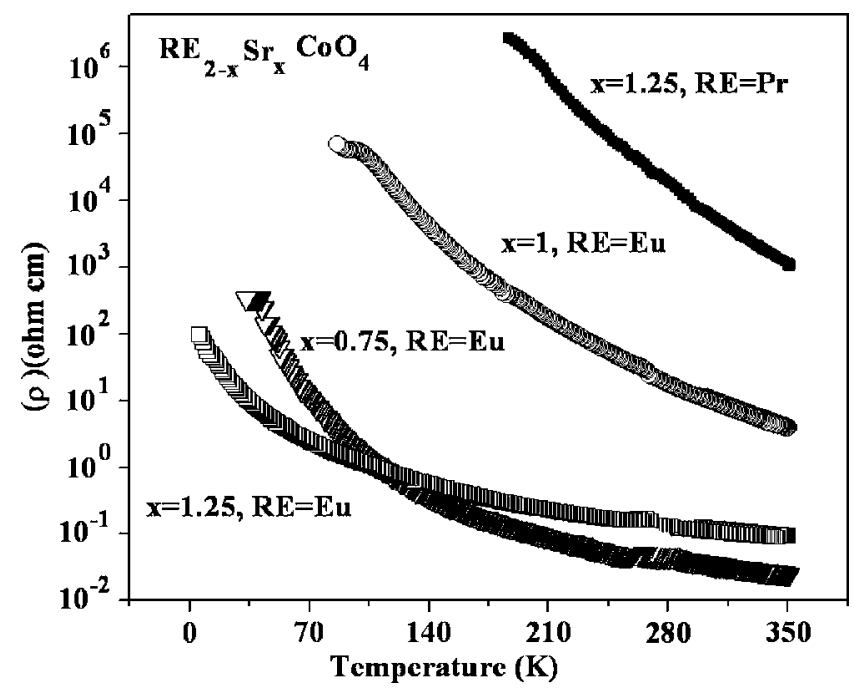

FIG. 3. Temperature dependence of the electrical resistivity.

The inverse molar susceptibility as a function of temperature in the range between 250 and $340 \mathrm{~K}$ for both systems was plotted (not shown here). We found that the data above $250 \mathrm{~K}$ can be well fitted to the Curie-Weiss law as indicated by the linear fitting. The linear fitting gives $\mu_{\text {eff }}$ $=5.23 \mu_{B}$ for $\operatorname{Pr}$ based system with $x>0.25$, and $\mu_{\text {eff }}$ $=2.63 \mu_{B}$ for Eu based system. For Pr based system with $x$ $<0.25$, the rest of the $\mu_{\text {eff }}$ values lay between the above mentioned two values. Taking into account the contributions from both $\mathrm{Eu}$ and Pr irons, the spin states of the $\mathrm{Co}^{2+}$ and $\mathrm{Co}^{3+}$ are estimated to be intermediate or high spin states in both $\mathrm{Eu}$ and Pr based systems. A detailed study on the assessment of spin states in correlation with the crystal field will be published elsewhere.

The temperature dependences of the resistivity for both $\mathrm{Pr}$ and Eu based samples are shown in Fig. 3. The Sr doping reduces the resistivity of the $\mathrm{Eu}$ based compounds significantly as compared to the Pr system. The room temperature resistivity for $\operatorname{Pr}_{0.75} \mathrm{Sr}_{1.25} \mathrm{CoO}_{4}$ is about $10 \mathrm{k} \Omega \mathrm{cm}$, while it is $10^{-1}$ and $10^{-2} \Omega \mathrm{cm}$ for the $\mathrm{Eu}_{1.25} \mathrm{Sr}_{0.75} \mathrm{CoO}_{4}$ and

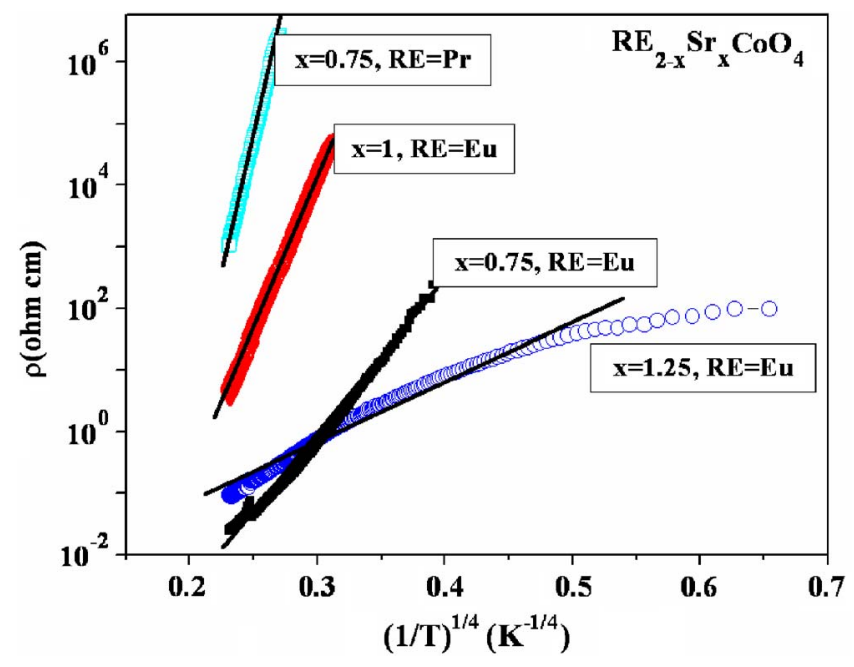

FIG. 4. (Color online) $\rho$ vs $(1 / T)^{1 / 4}$. 


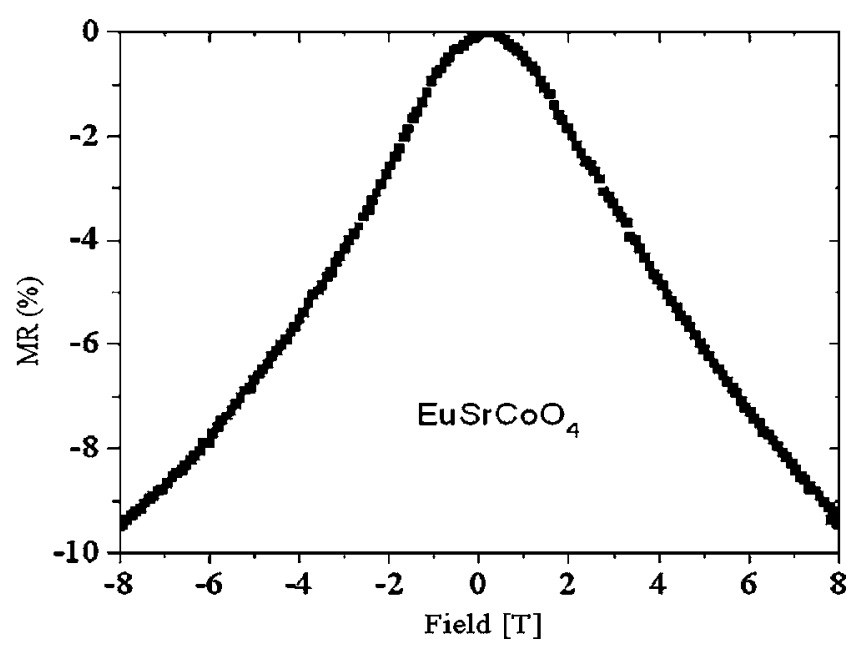

FIG. 5. The field dependence of magnetoresistance at $5 \mathrm{~K}$.

$\mathrm{Eu}_{0.75} \mathrm{Sr}_{1.25} \mathrm{CoO}_{4}$ samples, respectively. The $\mathrm{Eu}$ doped samples with $x<1$ have very low resistivity that is close to the resistivity of undoped $\mathrm{Sr}_{2} \mathrm{CoO}_{4}{ }^{2}$

The resistivity above $200 \mathrm{~K}$ for these samples shown in Fig. 3 can be well fitted by an equation $\rho=\rho_{\infty} \exp \left(T_{0} / T\right)^{1 / 4}$, as shown in Fig. 4 for $\left(T>200 \mathrm{~K},(1 / T)^{1 / 4}<0.27\right)$. This suggests that the three dimensional variable-range hopping (VRH) mechanism ${ }^{5}$ which has been proposed by Mott and observed in manganites compound ${ }^{6}$ can explain the electron conducting process in the Pr and Eu based compounds.

The field dependence of magnetoresistance of the $\mathrm{EuSrCoO}_{4}$ sample measured at $5 \mathrm{~K}$ is shown in Fig. 5. The MR value reaches $10 \%$ at $8 \mathrm{~T}$ which is larger than that of the pure polycrystalline $\mathrm{Sr}_{2} \mathrm{CoO}_{4}$ compounds. ${ }^{2}$ There is no clear hysteresis in the graph which is in agreement with the very small coercive field of this compound. This implies that the MR observed in this sample is due to the spin dependent scattering at grain boundaries.

The dielectric properties were studied for Pr based samples with $x=1$ and 0.75 , as shown in Fig. 6. The values of the dielectric constant $(\varepsilon)$ are over 2000 at low frequency less than $1 \mathrm{kHz}$ (not shown here) and gradually decrease with increasing frequencies. The $\varepsilon$ of the $x=1$ sample is greater than that of the $x=0.75$ sample, indicating that the charge induced capacitance in the $x=1$ sample is greater than that of the $x=0.75$ sample, this is in agreement with the trend of their resistivity measurements. The $\varepsilon$ remains above 50 at frequency up to $10 \mathrm{MHz}$ for the $x=1$ sample in contrast to about 10 at $10 \mathrm{MHz}$ for the $x=0.75$ sample. The dielectric loss $\delta$ drops down to 2-3 at a frequency of $1 \mathrm{MHz}$ and then
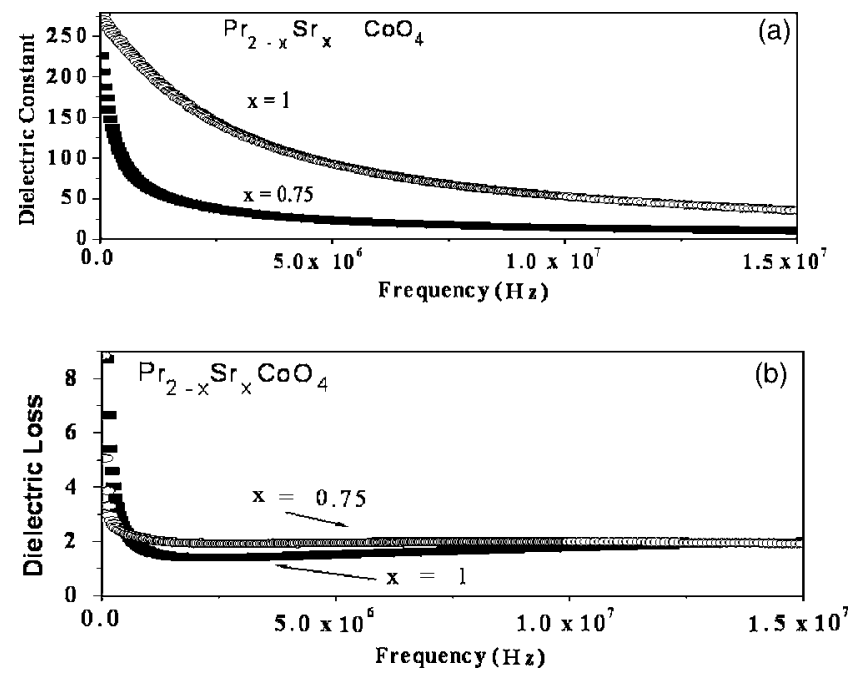

FIG. 6. Dielectric constant (a) and loss (b) of two $\mathrm{Sr}$ doped $\mathrm{Pr}_{2} \mathrm{CoO}_{4}$ samples.

increase very smoothly with increasing frequencies up to $10 \mathrm{MHz}$, indicating that extra conducting mechanism maybe responsible for such increase in $\delta$.

\section{CONCLUSION}

The $\mathrm{RE}_{2-x} \mathrm{Sr}_{x} \mathrm{CoO}_{4}(\mathrm{RE}=\mathrm{Pr}$ or $\mathrm{Eu})$ compounds crystallize in $\mathrm{K}_{2} \mathrm{NiF}_{4}$-type structures with space group $14 / \mathrm{mmm}$. Lattice parameters increase with $\mathrm{Sr}$ doping level. The compounds changed from paramagnetic to ferromagnetic with increasing Sr content and finally become typical ferromagnetic with $T_{C}$ of $230 \mathrm{~K}$ for the $\operatorname{Pr}$ based system with $x$ $=1.25$. Both systems changed from semiconductive to metallic with Sr content. Large dielectric constants with values of above 2000 are observed in low frequencies for samples with $x$ around 1 for Pr based compounds. The MR value of $10 \%$ is found in the $\mathrm{EuSrCoO}_{4}$ compounds.

\section{ACKNOWLEDGMENT}

This work is supported by funding from the Australian Research Council through ARC discovery project.

${ }^{1}$ K. Takada, H. Skurai, E. Takayama-Muromachi, F. Izumi, R. A. Dilanian, and T. Sasaki, Nature (London) 422, 53 (2003).

${ }^{2}$ X. L. Wang and E. Takayama-Muromachi, Phys. Rev. B 72, 064401 (2005).

${ }^{3}$ J. Matsuno, Y. Okimoto, Z. Fang, X. Z. Yu, Y. Matsui, N. Nagaosa, M. Kawasaki, and Y. Tokura, Phys. Rev. Lett. 93, 167202 (2004).

${ }^{4}$ B. A. Hunter, "Rietica: A Visual Rietveld Program," in Commission on Powder Diffraction Newsletter, Vol. 20, p. 21 (1998).

${ }^{5}$ N. F. Mott, J. Non-Cryst. Solids 1, 1 (1968).

${ }^{6}$ As a review, M. Ziese, Rep. Prog. Phys. 65, 143 (2002). 